\title{
Mupirocin - Are we in danger of losing it?
}

\author{
John M Conly $M D^{1}$, B Lynn Johnston $M D^{2}$
}

M upirocin (pseudomonic acid $A$ ) is one of four structurally related antibiotics, pseudomonic acids A, B, C and D, that were isolated originally from Pseudomonas fluorescens (1). It has a unique chemical structure that consists of a short fatty acid side chain linked via an ester bond to monic acid (2). Mupirocin inhibits RNA and protein synthesis by selectively binding to the bacterial isoleucyltRNA synthetase (IleS), preventing the formation of isoleucyl-tRNA, which, in turn, halts the incorporation of isoleucine into the nascent polypeptide chain (3). This mechanism of action is unique to mupirocin, and cross resistance between mupirocin and other antibiotics has not been reported (4).

Mupirocin is formulated as a $2 \%$ ointment preparation in a water-miscible polyethylene glycol base or as a cream preparation in a soft paraffin base. With direct application of mupirocin to the skin, mucous membranes or other tissues, very high local concentrations are achieved. There is negligible systemic absorption when mupirocin is applied topically (5). Application of the ointment, followed by the use of an occlusive dressing, enhances the penetration of mupirocin five- to 10-fold, but the amount that is absorbed is calculated to be less than $0.24 \%$ of the applied amount.

Once present in the stratum corneum, mupirocin is primarily eliminated via the upward movement and eventual desquamation of skin cells, rather than via metabolism (5).

Mupirocin is significantly more active in vitro in a weakly acid environment ( $\mathrm{pH}$ of 5.5 to 6 ) than at a $\mathrm{pH}$ of 8 , which may be important in the treatment of skin infections because the $\mathrm{pH}$ of skin is approximately 5.5 (6-8). The use of mupirocin includes the prophylaxis and treatment of primary and secondary infections of the skin, skin appendages and mucous membranes. In addition, mupirocin is used increasingly for the eradication of methicillin-resistant Staphylococcus aureus (MRSA), an indication that was not intended originally.

Mupirocin is active primarily against Gram-positive organisms. It is bactericidal against $S$ aureus and Staphylococcus epidermidis, including methicillin-resistant and other antibiotic-resistant strains at concentrations that are achieved with topical application $(6,8)$. It is also readily bactericidal against several Streptococcus species, including Streptococcus pyogenes, Streptococcus agalactiae and Streptococcus viridans, but is inactive against anaerobic streptococci and enterococci. Mupirocin has no activity against Gramnegative organisms, anaerobes and fungi, and exhibits minimal activity against normal skin flora such as Micrococcus, Corynebacterium and Propionibacterium species (6).

Definitions of mupirocin resistance vary and no National Committee for Clinical Laboratory Standards guidelines exist for topical agents, but most studies have recognized low-level (minimal inhibitory concentration [MIC] of $8 \mathrm{mg} / \mathrm{L}$ to $256 \mathrm{mg} / \mathrm{L}$ ) and high-level (MIC of $512 \mathrm{mg} / \mathrm{L}$ or greater) resistance (4). Low-level resistance is thought to arise from point mutations in the chromosomally encoded IleS gene. This resistance is considered stable and nontransferable (4). The development of high-level mupirocin resistance results from the acquisition of a plasmid that contains the mupA resistance element, which contains a modified IleS-2 gene $(9,10)$. Such isolates typically carry two

\footnotetext{
${ }^{1}$ Departments of Pathology and Laboratory Medicine, Medicine, and Microbiology $\mathbb{E}$ Infectious Diseases, University of Calgary, Calgary, Alberta; ${ }^{2}$ Queen Elizabeth II Health Sciences Centre and Dalhousie University, Halifax, Nova Scotia

Correspondence and reprints: Dr John Conly, Departments of Pathology and Laboratory Medicine, Medicine, and Microbiology $\mathcal{E}$ Infectious Diseases, Centre for Antimicrobial Resistance, 1638-10th Avenue SW, Calgary, Alberta T3C 0J5. Telephone 403-209-5338,

fax 403-209-5347, e-mail jconly@ucalgary.ca and Dr Lynn Johnston, Room 5014 ACC, Queen Elizabeth II Health Sciences Centre,

1278 Tower Road, Halifax, Nova Scotia B3H 2Y9. Telephone 902-473-8477, fax 902-473-7394, e-mail ljohnsto@is.dal.ca
} 
distinct IleS-2 genes: the constitutive chromosomally based gene that may, itself, encode for variable levels of mupirocin resistance, and the acquired high-level resistance plasmid-based gene $(10,11)$. Farmer et al (12) showed that the antibiotic concentration that halved isoleucyltRNA synthetase activity correlated well with the MIC of $3.3 \times 10^{-2} \mathrm{mg} / \mathrm{L}$ for mupirocin-susceptible strains, $1.3 \times 10^{-1}$ $\mathrm{mg} / \mathrm{L}$ for low-level resistant strains, and $7.5 \mathrm{mg} / \mathrm{L}$ in highlevel resistant strains (12). The origin of mupA is not known, but it has been found in strains of staphylococci that existed before the release of mupirocin (4), and may have a natural reservoir. Low-level mupirocin-resistant organisms may be induced in vitro and were observed in an early in vivo study (4). Low-level mupirocin resistance is not considered to be significant clinically, given that the concentration of mupirocin in the $2 \%$ ointment exceeds $20,000 \mathrm{mg} / \mathrm{L}(4,13)$. However, clinical failure due to highlevel mupirocin resistance (MIC of $512 \mathrm{mg} / \mathrm{L}$ or greater) is well recognized.

Cookson (4) suggested that increasing reports of highlevel mupirocin resistance in staphylococci may limit the effectiveness of this agent in the future, particularly for the control of MRSA. Prolonged use and multiple courses of mupirocin seem to be the factors that are associated most frequently with the development of mupirocin resistance. Long term use of mupirocin was first reported to lead to the development of irreversible resistance in staphylococci over a decade ago $(14,15)$, and has been reported in several parts of the world, including Europe $(4,16,17)$, Australia (18) and the Americas $(19,20)$. Unfortunately, not all of these reports have distinguished high-level from low-level resistance, but interpretive criteria for correlating inhibitory zone diameters with MICs based on agar dilution or E test strips $(21,22)$ may provide some guidance in interpreting these reports.

Some of the reports of the development of mupirocin resistance are striking in the magnitude of increase in percentage of resistant isolates that has been noted over time. Miller et al (19) reported an increase in mupirocin resistance among MRSA isolates from 2.7\% in 1990 to $65 \%$ in 1993. Almost $75 \%$ of the isolates demonstrated no discernible zone of inhibition, which suggests that most of the isolates possessed high-level resistance. The increase in mupirocin resistance was associated with the widespread use of mupirocin for the decolonization of patients during an MRSA epidemic in this Canadian institution (19). In two hospitals in Brazil, a similar pattern was noted, with an overall prevalence of mupirocin resistance of $63 \%$ (with $61 \%$ exhibiting high-level resistance) in the facility that used mupirocin on a daily basis for colonized MRSA patients, compared with $6 \%$ in the facility where mupirocin was used rarely (23). At a Veteran Affairs medical centre in the United States, a significant temporal increase in mupirocin resistance was noted over three years (24) among strains of MRSA on a background of high mupirocin usage. The prevalence of high-level mupirocin resistance was $42 \%$ in this centre. This latter report also included a case control study that showed that the presence of a decubitus ulcer correlated with high-level mupirocin resistant isolates of $S$ aureus. In Warsaw, Poland, an outbreak of mupirocin-resistant staphylococci occurred on two wards of a large teaching hospital after the introduction of the use of mupirocin for the treatment of skin infections (25). Over a 17-month period, 53 mupirocin-resistant isolates of S aureus, S epidermidis, Staphylococcus haemolyticus, Staphylococcus xylosus and Staphylococcus capitis were identified, representing $19.5 \%$ of all staphylococcal isolates that were identified in the two wards during that time. The majority $(87 \%)$ of the isolates were found to harbour highlevel resistance to mupirocin. Almost all of the isolates were also resistant to methicillin. Although the $S$ aureus isolates were found to represent a single epidemic clone, the $S$ epidermidis population was much more diverse. Of note, six isolates of $S$ epidermidis were demonstrated to express both low- and high-level resistance mechanisms simultaneously - the first identification of such dual mupirocin-resistant phenotype-bearing strains. In Japan, no isolates of methicillin-susceptible $S$ aureus or MRSA collected from 43 hospitals nationwide in 1993 were found to have mupirocin resistance. However, following the introduction of intranasally administered mupirocin, mupirocin resistance was detected in $5.3 \%$ of MRSA strains and $23.3 \%$ of coagulase-negative staphylococci (Staphylococcus hominis, S epidermidis, S chromogenes) that were collected from the nares of patients over four years (26).

Mupirocin has been used extensively to prevent $S$ aureus infections in patients undergoing peritoneal dialysis. Recently, two reports described the emergence of high-level resistance to mupirocin in both methicillin-susceptible and methicillin-resistant strains of $S$ aureus in patients undergoing chronic peritoneal dialysis. Perez-Fontan et al (27) reported the emergence of MRSA in peritoneal dialysis patients and their partners over 10 years of using topical mupirocin. From 1990 to 1996, no high-level mupirocin resistance was noted. Mupirocin resistance subsequently developed and increased to 8.3\% between 1997 and 1998, and to $12.4 \%$ between 1999 and 2000. Resistance was associated frequently with repeated mupirocin treatments for recolonization. The cumulative incidence of $S$ aureus exit site infection from 1997 to 2000 was $32.3 \%$ in patients who were colonized by MRSA compared with $14.5 \%$ in those patients who were colonized by mupirocin-sensitive strains, which suggested that resistance has significant clinical impact. In addition, Annigeri et al (28) reported the significant emergence of high-level resistance to $S$ aureus after a four-year use of mupirocin as prophylaxis for exit site infections. Of all the $S$ aureus isolates collected in a point prevalence survey four years after the initiation of the use of mupirocin, $15 \%$ were found to have high-level resistance to mupirocin, which was significantly increased compared with a similar survey after one year's use, when no resistance was 
detected. None of the isolates were resistant to methicillin in this study (28).

The emergence of high-level resistance to mupirocin in both outbreak and nonoutbreak settings, and among different patient populations, is a cause for concern. Collectively, these studies suggest that prolonged and widespread use of mupirocin is associated with the development of resistance. The spread of this resistance may occur through horizontal transfer of microorganisms carrying mupA, or through

\section{REFERENCES}

1. Ward A, Campoli-Richards DM. Mupirocin. A review of its antibacterial activity, pharmacokinetic properties and therapeutic use. Drugs 1986;32:425-44.

2. Parenti MA, Hatfield SM, Leyden JJ. Mupirocin: A promising new topical antimicrobial agent. J Antimicrob Chemother 1987;6:761-70.

3. Hughes J, Mellows G. Inhibition of isoleucyl-transfer ribonucleic acid synthetase in Escherichia coli by pseudomonic acid. Biochem J 1978;176:305-18.

4. Cookson BD. The emergence of mupirocin resistance: A challenge to infection control and antibiotic prescribing practice.

J Antimicrob Chemother 1998;41:11-8.

5. Baines PJ, Jackson D, Mellows G, Swaisland AM, Tasker TCG. Mupirocin: Its chemistry and metabolism. In: Wilkinson JD and Price JD, eds. Mupirocin - A Novel Topical Antibiotic. London: Royal Society of Medicine, 1984:13-22.

6. Sutherland R, Boon RJ, Griffin KE, et al. Antibacterial activity of mupirocin (pseudomonic acid), a new antibiotic for topical use. Antimicrob Agents Chemother 1985;27:495-8.

7. Casewell MW, Hill RLR. The laboratory assessment of the antistaphylococcal activity of mupirocin. In: Wilkinson JD and Price JD, eds. Mupirocin - A Novel Topical Antibiotic. London: Royal Society of Medicine, 1984:57-64.

8. Casewell MW, Hill RLR. In vitro activity of mupirocin (pseudomonic acid) against clinical isolates of Staphylococcus aureus. J Antimicrobial Chemother 1985;15:523-31.

9. Woodford N, Watson AP, Patel S, Jevon M, Waghorn DJ, Cookson BD. Heterogeneous location of the mupA high-level mupirocin resistance gene in Staphylococcus aureus. J Med Microbiol 1998;47:829-35.

10. Thomas DG, Wilson JM, Day MJ, Russell AD. Mupirocin resistance in staphylococci: Development and transfer of isoleucyl-tRNA synthetase-mediated resistance in vitro. J Applied Microbiol 1999;86:715-22.

11. Gilbart J, Perry CR, Slocombe B. High-level resistance in Staphylococcus aureus: Evidence for two distinct isoleucyl-tRNA synthetases. Antimicrob Agents Chemother 1993;37:32-8.

12. Farmer TH, Gilbart J, Slocombe B. Biochemical basis for resistance in strains of S aureus. J Antimicrob Chemother 1992;30:587-96.

13. Bradley SF. Effectiveness of mupirocin in the control of methicillinresistant Staphylococcus aureus. Infect Med 1993;10:23-31.

14. Baird D, Coia J. Mupirocin-resistant Staphylococcus aureus. Lancet 1987;2:387-8.

15. Smith MD, Sanghrijka M, Lock S. Mupirocin-resistant Staphylococcus aureus. Lancet 1987;2:1472-3.

16. Alarcon T, Sanz JC, Blanco F, Domingo D, Lopez-Brea M. High- transfer of the mupA containing plasmid in both coagulasepositive and coagulase-negative staphylococci. With rising rates of MRSA in Canada, the judicious use of topical mupirocin cannot be overemphasized. In addition, in settings where mupirocin is used to any significant degree, reliable methods to determine and monitor resistance to this agent should be implemented by microbiology laboratories to provide an early warning system to facilitate interventions to minimize its spread.

level mupirocin resistance among Spanish methicillin-resistant Staphylococcus aureus. Eur J Clin Microbiol Infect Dis 1998;17:877-9.

17. Schmitz FJ, Lindenlauf E, Hofmann B, et al. The prevalence of low-and high-level mupirocin resistance in staphylococci from 19 European hospitals. J Antimicrob Chemother 1998;42:489-95.

18. Riley TB, Carson CF, Bowman RA, et al. Mupirocin-resistant methicillin-resistant Staphylococcus aureus in Western Australia. Med J Australia 1994;161:397-8.

19. Miller MA, Dascal A, Portnoy J, Mendelson J. Development of mupirocin resistance among methicillin-resistant Staphylococcus aureus after widespread use of nasal mupirocin ointment. Infect Control Hosp Epidemiol 1996;17:811-3.

20. Ramos RL, Teixeira LA, Ormonde LR, et al. Emergence of mupirocin resistance in multiresistant Staphylococcus aureus clinical isolates belonging to Brazilian epidemic clone III:B:A. J Med Microbiol 1999;48:303-7.

21. Cercenado E, Padilla B, Garcia-Garrote F, et al. High incidence of mupirocin-resistant and -susceptible Staphylococcus aureus. The 38th Interscience Conference on Antimicrobial Agents and Chemotherapy, San Diego, September 24 to 27, 1998. (Abst C-75)

22. Finlay JE, Miller L, Poupard JA. Interpretive criteria for testing susceptibility of staphylococci to mupirocin. Antimicrob Agents Chemother 1997;41:1137-9.

23. Katia R, dos Santos N, de Souza F, et al. Emergence of high-level mupirocin resistance in methicillin-resistant Staphlyococcus aureus isolated from Brazilian university hospitals. Infect Control Hosp Epidemiol 1996;17:813-6.

24. Vasquez JE, Walker ES, Franzus BW, et al. The epidemiology of mupirocin resistance among methicillin-resistant Staphlyococcus aureus at a Veteran's Affairs hospital. Infect Control Hosp Epidemiol 2000;7:459-64.

25. Leski TA, Gniadkowski M, Skoczynska A, et al. Outbreak of mupirocin-resistant staphylococci in a hospital in Warsaw, Poland due to plasmid transmission and clonal spread of several strains. J Clin Microbiol 1999;37:2781-8.

26. Nakagawa S, Iinuma Y, Yamamoto $H$, et al. Detection of mupirocin reistant staphylococci from patients treated with mupirocin. Kansenshogaku Zasshi 2001;75:7-13.

27. Perez-Fontan M, Rosales M, Rodriguez-Carmona A, et al. Mupirocin resistance after long-term use for Staphylococcus aureus colonization in patients undergoing chronic peritoneal dialysis. Am J Kid Dis 2002;39:337-41.

28. Annigeri R, Conly J, Vas S, et al. Emergence of mupirocin-resistant Staphylococcus aureus in chronic peritoneal dialysis patients using mupirocin prophylaxis to prevent exit-site infections. Perit Dial Int 2001;21:554-9. 


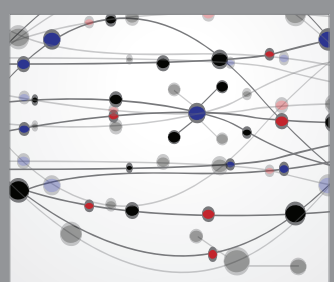

The Scientific World Journal
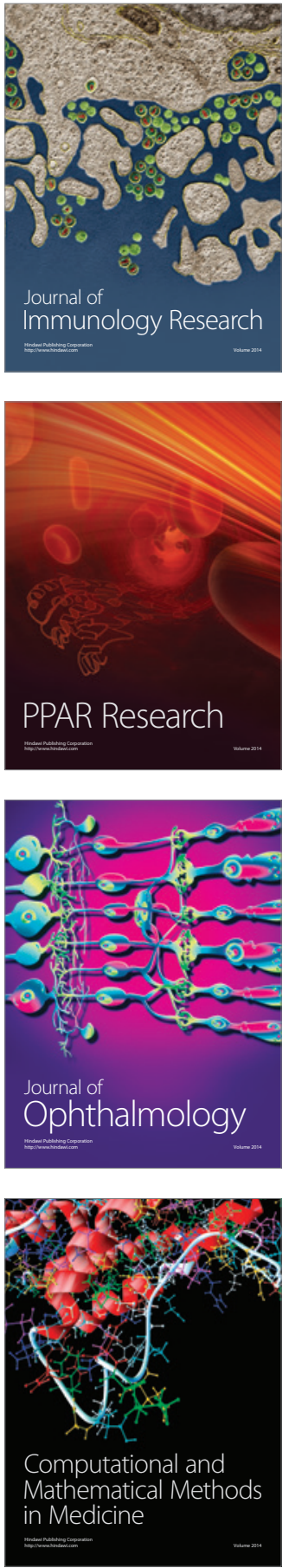

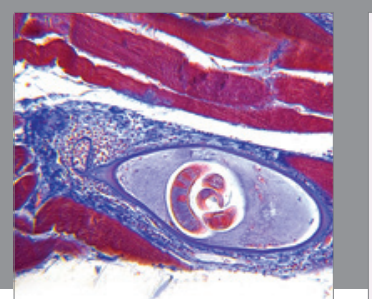

Gastroenterology Research and Practice

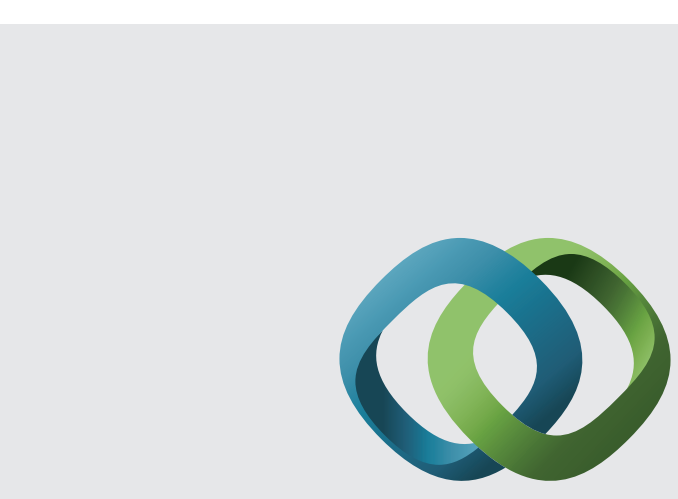

\section{Hindawi}

Submit your manuscripts at

http://www.hindawi.com
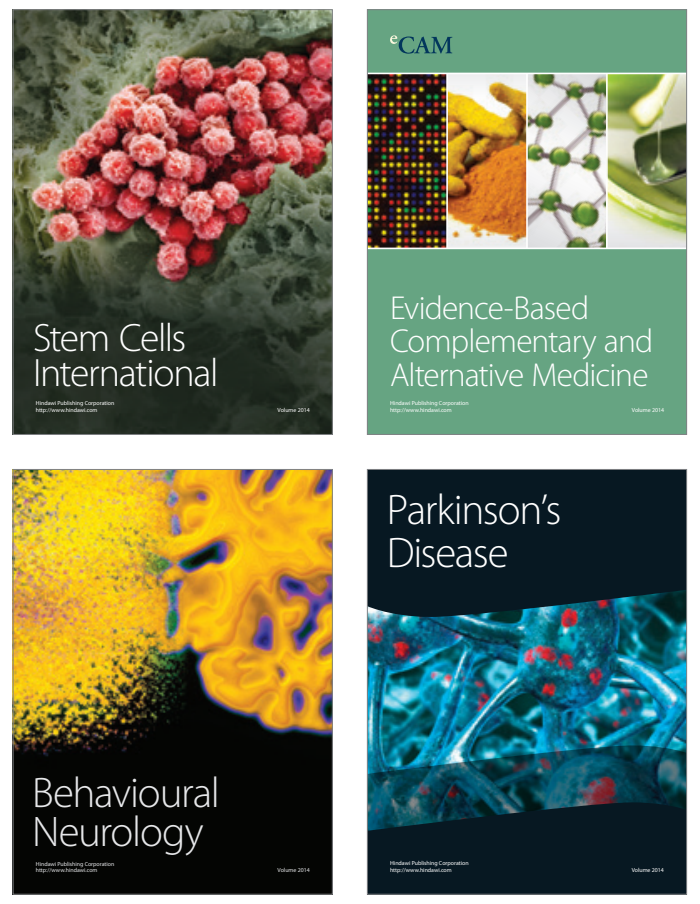
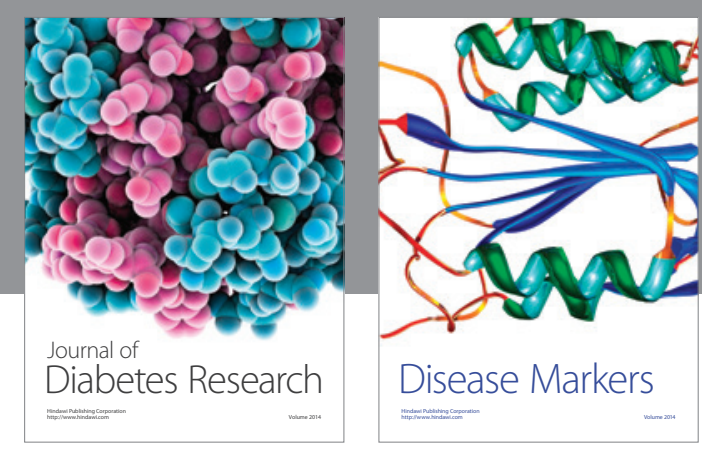

Disease Markers
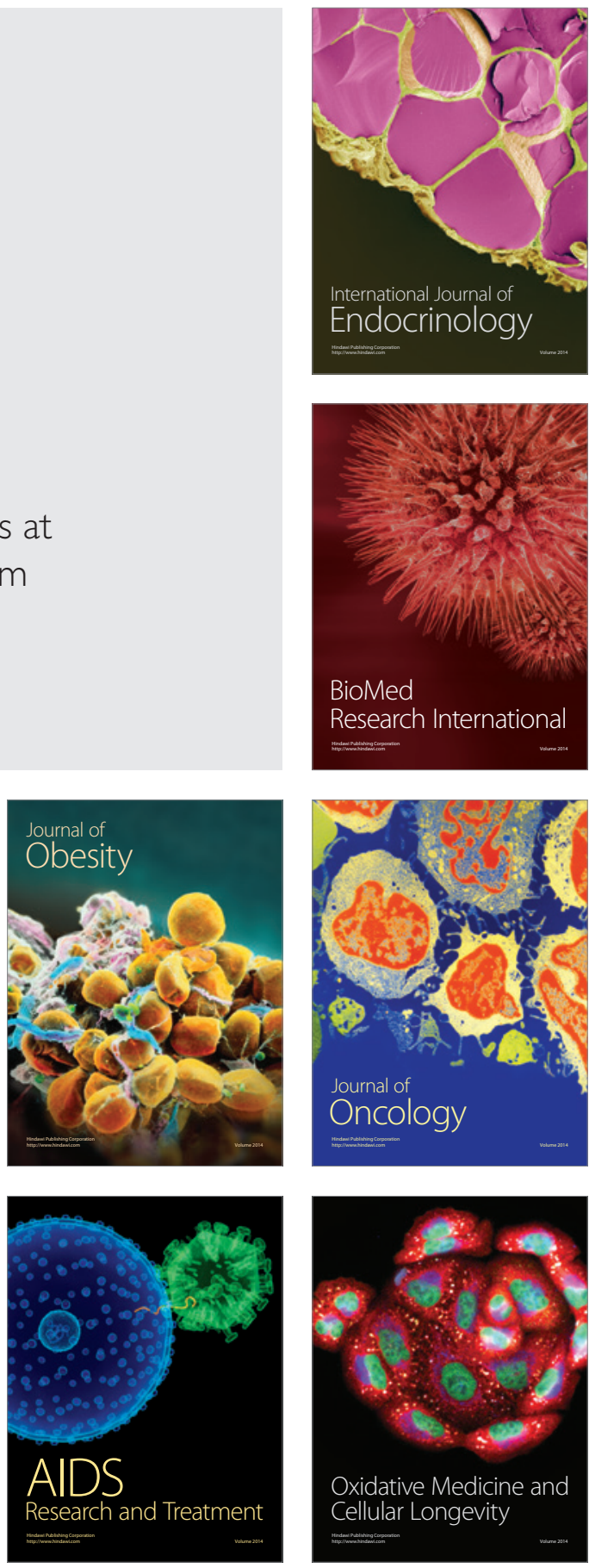